CUADERNOS DE ESTUDIOS GALLEGOS, LXII Núm. 128 (enero-diciembre 2015), págs. 281-303

ISSN: $0210-847 \mathrm{X}$

DOI: 10.3989/ceg.2015.128.09

\title{
TRAS LA HUELLA DEL DESCONOCIDO MAESTRO CIEGO DEL COLEGIO DE SORDOMUDOS Y CIEGOS \\ DE SANTIAGO: JUAN LORENZO GONZÁLEZ (FINALES DEL SIGLO XIX)
}

\author{
Ana Rodríguez Díaz \\ Elisa Gavari Starkie \\ Universidad Nacional de Educación a Distancia (UNED)
}




\section{TRAS LA HUELLA DEL DESCONOCIDO MAESTRO CIEGO DEL COLEGIO DE SORDOMUDOS Y CIEGOS DE SANTIAGO: JUAN LORENZO GONZÁLEZ (FINALES DEL SIGLO XIX) RESUMEN}

La difusión del sistema Braille como un medio de comunicación alternativo al visual ha supuesto un factor determinante en la integración de las personas ciegas. El sistema Braille se introdujo en Argentina mediante la enseñanza de Juan Lorenzo González. Este artículo pretende contribuir al conocimiento de este ignorado maestro ciego. A pesar de que en España permaneció eclipsado por otros pedagogos, su personalidad altruista le llevó a instalarse en la ciudad de Buenos Aires, donde desarrolló su mayor obra pedagógica. El 1 de marzo de 1887, recién llegado a dicha ciudad, la dirigente de la Sociedad de Beneficencia de Buenos Aires propuso al pedagogo español como profesor de ciegos en el Asilo de Huérfanos. La propuesta se aceptó con una rápida respuesta, y unos meses después de su nombramiento comenzó su tarea. Fue entonces cuando se inició la atención institucional y profesional de los ciegos en Argentina; hasta aquella fecha los ciegos solo habían recibido sustento material y amparo caritativo. En este estudio, abordado desde una perspectiva histórica y fundamentado en fuentes archivísticas, prensa y publicaciones históricas y actuales (principalmente argentinas), se aportan datos significativos que permiten desvelar algunas de las incógnitas sobre la biografía de esta destacada personalidad.

Palabras Clave: Braille, enseñanza de ciegos, educación especial en América Latina, Colegio de sordomudos y de ciegos de Santiago de Compostela, Juan Lorenzo González.

\section{TRAS A PEGADA DO DESCOÑECIDO MESTRE CEGO DO COLEXIO DE XORDOMUDOS E CEGOS DE SANTIAGO: JUAN LORENZO GONZÁLEZ (FINAIS DO SÉCULO XIX) \\ Resumo}

A difusión do sistema Braille como un medio de comunicación alternativo ao visual supuxo un factor determinante na integración das persoas cegas. O sistema Braille introduciuse en Arxentina mediante o ensino de Juan Lorenzo González. Este artigo pretende contribuír ao coñecemento deste ignorado mestre cego. A pesar de que en España permaneceu eclipsado por outros pedagogos, a súa personalidade altruísta levouno a instalarse na cidade de Bos Aires, onde desenvolveu a súa maior obra pedagóxica. O $1{ }^{\circ}$ de marzo de 1887, recén chegado á devandita cidade, a dirixente da Sociedade de Beneficencia de Bos Aires propuxo o pedagogo español como profesor de cegos no Asilo de Orfos. A proposta foi aceptada cunha rápida resposta e, uns meses despois do seu nomeamento, comezou a súa tarefa.

Foi neste momento cando se iniciou a atención institucional e profesional dos cegos en Arxentina; ata entón os cegos só recibiran sustento material e amparo caritativo.Neste estudo, abordado dende unha perspectiva histórica fundamentado en fontes arquivísticas, prensa e publicacións históricas e actuais, principalmente arxentinas, achéganse datos significativos que permiten desvelar algunhas das incógnitas sobre a biografía desta destacada personalidade.

Palabras Clave: Braille,ensino de cegos, educación especial en América Latina, Colexio de sordomudos y de cegos de Santiago de Compostela, Juan Lorenzo González.

\section{ON THE TRAIL OF THE UNKNOWN SPANISH BLIND TEACHER FROM THE COLLEGE FOR THE DEAF AND BLIND IN SANTIAGO: JUAN LORENZO GONZÁLEZ (IN THE LATE $19^{\mathrm{TH}}$ CENTURY) ABSTRACT}

Dissemination of Braille as an alternative means of visual communication has been a key factor in the integration of blind people. The Braille system was introduced in Argentina by teaching Juan Lorenzo González.This article aims to contribute to the knowledge of the unknown blind master. Although he remained in Spain overshadowed by other teachers, his altruistic personality led him to settle in the city of Buenos Aires, where he developed his greatest educational work. On 1 March 1887, the official of the Sociedad de Beneficencia of Buenos Aires proposed to him, a newcomer to this city, as a teacher of the blind people in the Asilo de Huérfanos. The proposal was accepted with a quick response and a few months after his appointment, he began his task. It was then when the institutional and professional care of the blind people in Argentina began; until then had only the blind received material support and charitable shelter. This study was approached from a historical perspective, has been based on archival sources,historical and current newspapers and publications, mainly Argentina. In this research reveal meaningful data that allow some of the unknowns about the biography of this remarkable personality.

KEY wORDS: Braille, teaching of blind people, special education in Latin America, of college for the deaf and blind in Santiago de Compostela, Juan Lorenzo González. 
Recibido/Received: 31/01/2015

Aceptado/Accepted: 10/08/2015

El Sistema BRAILLE: MÉTOdO UNIVERSAL DE LECTURA Y ESCRITURA EN LOS PRIMEROS COLEGIOS E INSTITUCIONES DE CIEGOS DE FINALES DEL XIX

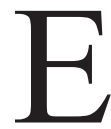

$\mathrm{n}$ la sociedad medieval europea, la discriminación ante los que presentaban deficiencias físicas o psíquicas se manifestaba en ofertarles la mendicidad como único recurso de subsistencia. La ocupación más frecuente de los ciegos era pedir limosna: "El ciego, en la cultura medieval, es el mendigo por antonomasia". Lo cierto es que los privados del sentido de la vista encontraban más dificultades a la hora de emprender cualquier actividad laboral que los que padecían otro tipo de deficiencias. Además, como señala Garvía Soto (1992), los ciegos eran más proclives a recibir limosna. La ceguera provocaba en las gentes un sentimiento compasivo, mayor que otras enfermedades que igualmente obligaban a la mendicidad. Por otra parte, Montoro Martínez (1995) afirma que existía la convicción de que la asistencia caritativa era una solución más económica y menos problemática que la que suponía integrar a los ciegos en la sociedad mediante su educación. La instrucción de un ciego no era igual que la de un vidente, y requería profesorado y material especial.

Durante muchos siglos la humanidad ha practicado la caridad con los faltos de vista, pero no se ha preocupado de enseñarles a ganarse el diario sustento y les ha negado un puesto de trabajo remunerado, porque el efecto de la acción caritativa es más inmediato y no entraña responsabilidad ni problemas, en tanto que la instrucción de un invidente exige tiempo, dinero y determinadas aptitudes en maestro y discípulo ${ }^{2}$.

\footnotetext{
1 Carmen López Alonso, La pobreza en la España medieval, Madrid, Ministerio de Trabajo y Seguridad Social, 1986, pág. 51.

2 Jesús Montoro Martínez, Los ciegos en la historia, vol. 5, Madrid, ONCE, 1995, pág. 13.
} 
Existía también la opinión de que los conocimientos adquiridos con la enseñanza nunca podrían procurar a los ciegos los medios convenientes para subsistir ${ }^{3}$. De ahí que su educación fuese considerada casi como un lujo innecesario. Si bien estas ideas perduraron más allá del siglo XIX, la mentalidad empirista ilustrada que imperaba en el siglo XVIII abordó de manera mucho más optimista el problema de la enseñanza de los invidentes, explorando diferentes métodos para facilitar su instrucción, compensando su deficiencia visual con el uso de los otros sentidos. Este es el concepto de instrucción que sostenían pedagogos y maestros, como Valentin Haüy, que creó en Francia la primera escuela para ciegos, la Institution National des Jeunes Aveugles ${ }^{4}$. Valentín Haüy ideó un procedimiento para la lectura y la escritura de los ciegos que trataba de equiparar en lo posible la educación de estos a los niveles y procedimientos seguidos en la educación de los normovisuales.

Otro francés, Charles Barbie, capitán de artillería, dio un nuevo impulso a la instrucción de los invidentes con un modelo de escritura nocturna, pensado para el ejército, y que debía leerse al tacto. En el año 1821, Barbie presentó su modelo de escritura en la escuela de París. Louis Braille, alumno de la escuela, se mostró interesado en este sistema, y fundamentándose en este modelo de escritura, desarrolló uno nuevo mucho más práctico. Este sistema resultaba fácilmente legible con el dedo, en comparación con otros alfabetos de caracteres latinos en relieve destinados a la lectura táctil utilizados con anterioridad. Además, se adaptaba con facilidad al lenguaje musical, al matemático y a cualquier lengua.

Este modelo de escritura, llamado "braille" en homenaje a su creador, consiguió imponerse a otros métodos, y fue, de 1860 a 1880, el sistema adoptado en toda Europa en su forma original, a excepción de ciertas modificaciones necesarias para adaptarse a los distintos alfabetos visuales ${ }^{5}$. En el Primer Congreso Internacional de Sordomudos y Ciegos, que se celebró en París en el año 1879, se adoptó oficialmente el sistema braille para la enseñanza de las personas ciegas como sistema universal. Sin embargo, como afirma Burgos Bordonau ${ }^{6}$ (2004), no es fácil dar una fecha exacta del momento en el que este sistema se empezó a emplear como único método de lectura y escritura en colegios e instituciones de ciegos. En España, el sistema braille se introdujo en el año 1840. A partir de la Ley de Instrucción Pública de 1857, la legislación española asumió lo relativo

\footnotetext{
3 Juan Manuel Ballesteros y Francisco Fernández Villabrille, Curso elemental de instrucción de ciegos, Madrid, Imprenta del Colegio Nacional de Sordomudos, 1862, pág. 120.

4 Antonio Aguado díaz, Historia de las deficiencias, Madrid, Once, 1995, pág. 103.

5 Clutha N. Mackenzie, La escritura Braille en el mundo, París, Unesco, 1954, pág. 21.

6 Esther Burgos Bordonau, Historia de la enseñanza musical para ciegos en España, 1830-1938, Madrid, ONCE, 2004.
} 
a esta educación especial, y comenzó la creación de instituciones para los niños sordomudos y ciegos.

La educación de las personas ciegas en América Latina se inició también con el sistema Braille. En el siglo XIX se había extendido solamente a cuatro países de América Latina: Brasil, México, Cuba y Argentina ${ }^{7}$ En Argentina, al igual que en el resto de América Latina, no hubo otro sistema de lectoescritura, y se dio simultáneamente la llegada del braille con el comienzo de la alfabetización y educación, la instalación de imprentas y de bibliotecas. En 1884 se recibieron en Buenos Aires los dos primeros libros en braille: un manual de geometría con algunas ilustraciones y otro de lecturas escolares. La señora Ana M. ${ }^{a}$ del Campillo Pedriel, presidenta de la Sociedad de Beneficencia, los había enviado desde París para que el escaso número de ciegos con que contaba la Casa de Expósitos pudiese hacer uso de ellos. Al año siguiente, un brote de viruela obligó a trasladar a los niños ciegos al Asilo de Huérfanos, organizando un departamento especial para ellos con el fin de proceder a su instrucción. "Este sería el antecedente más temprano de la actividad tiflológica en la República Argentina"8. En el año 1887, cuando se inició la educación de los niños ciegos en Argentina, en España ya llevaban más de veinte años funcionando algunos colegios y escuelas en ciudades como Barcelona, Madrid, Santiago de Compostela o Burgos, dedicados a esta enseñanza especial.

Sin embargo, en la década de los treinta del siglo XX, según recoge un artículo del diario El pueblo gallego ${ }^{9}$ firmado por Emilio Arroyo Fuentes, profesor del Colegio de Sordomudos y Ciegos de Santiago de Compostela, el estado de abandono en que se hallaba la enseñanza de los ciegos en España era lamentable. Algunas repúblicas hispanoamericanas se hallaban mucho más adelantadas en esta materia que España. Este fue el caso de Argentina, donde se realizó una gran obra tiflológica que culminó con la gran Biblioteca Argentina para ciegos, con una excepcional imprenta y biblioteca braille para todos los ciegos de habla española. En las primeras décadas del siglo XX Argentina se situaba en un plano muy superior a España en el desarrollo de esta enseñanza especial. Y, como testimonia el periódico citado anteriormente:

\footnotetext{
7 Judith A. Varsavsky, "El movimiento asociativo de los ciegos en América Latina", Cadernos GESTA, 1 (julho 2001) [en línea], disponible en <http://www.gesta.org/gesta01/> [Consulta: 12/06/ 2014].

8 Angel Prignano, Una Historia de Relieve. Biblioteca Argentina para ciegos. 1924-2004. Ochenta años de servicio, Buenos Aires, Biblioteca Argentina para Ciegos, 2004, pág. 9.

9 “Compostela al día. La enseñanza y los ciegos", El pueblo gallego (6 de noviembre de 1931), pág. 10.
} 
...precisamente recibió la enseñanza de manos de un español, el gallego, Juan Lorenzo González, profesor que fue en el Colegio de Santiago, fue quien fundó en Buenos Aires la primera escuela para ciegos.

El Colegio regional de Santiago de Compostela, pionero y referente en la EDUCACIÓN ESPECIAL DE SORDOMUDOS Y CIEGOS EN ESPAÑA. JUAN LORENZO GONZÁLEZ, UN ALUMNO DESTACADO

La educación española en las últimas décadas del siglo XIX presentaba un enorme atraso. El analfabetismo alcanzaba, a mediados del siglo XIX, aproximadamente al $75 \%$ del total de la población. Los planteamientos educativos, lo mismo que sucedía con el resto de asuntos gubernamentales, se movían entre la tradición y la reforma, según quien ocupase el cargo en el gobierno (conservadores o liberales). En países europeos industrializados como Alemania o Suiza la imagen de la escuela iba cambiando gracias a un nuevo espíritu reformista, mientras que en España la modernización de la enseñanza llegaría bastante más tarde ${ }^{10}$. La aprobación de la Ley de Instrucción Pública del 9 de septiembre de 1857, conocida como "Ley Moyano", es lo más destacable dentro del panorama educativo en España en este periodo. El objetivo de esta centenaria Ley de Instrucción Pública era solucionar el problema educativo, apostando, de acuerdo con la ideología de un liberalismo moderado, por el centralismo, la uniformidad y la secularización parcial. También apostaba por la instrucción primaria obligatoria y gratuita, a condición de figurar como pobre de solemnidad. Tras la promulgación de dicha Ley, el Estado quedaría también obligado a educar a ciegos y sordomudos, cosa que suponía un acontecimiento fundamental para la futura integración social de estos alumnos. En su artículo 6 se recoge lo siguiente: "La primera enseñanza se dará, con las modificaciones convenientes, a los sordomudos y ciegos en los establecimientos esenciales que hoy existen y en los demás que se crearán con este objeto" "El artículo 108 añadía la conveniencia de establecer un establecimiento de enseñanza especial en cada distrito:

Promoverá asimismo el Gobierno las enseñanzas para los sordomudos y ciegos, procurando que haya por lo menos una Escuela de esta clase en cada Distrito universitario y que en las

\footnotetext{
${ }^{10}$ James Bowen, Historia de la Educación Occidental, vol. 3: El occidente moderno, Barcelona, Herder, 1992, pág 550.

${ }^{11}$ Francisco Fernández Villabrille, Estado actual y organización de la enseñanza de Sordomudos y ciegos. Memoria dirigida al Ministro de Fomento, Madrid, Imprenta del Colegio de Sordomudos, 1862, pág. 87.
} 
públicas de niños se atienda, en cuanto sea posible á la educación de aquellos desgraciados ${ }^{12}$.

En cuanto a los distritos universitarios a los que hace mención el artículo, hay que remontarse al Plan General de estudios aprobado el 17 de septiembre de 1845, en el que el ministro de la gobernación, Pedro José Pidal y Carniado, redujo las universidades de España a diez (artículo 67) y dividió el territorio de la España peninsular e islas adyacentes en tantos distritos cuantas universidades (artículo 138). Entre estos distritos, figuraba el de Santiago de Compostela.

El rector de la Universidad, el Excmo. Sr. D. Juan Viñas, solicitó al gobierno central la creación de una de estas escuelas para Santiago, en base a los arts. 6 y 108 de la Ley de Instrucción pública de 1857, en los que se establecían las escuelas de enseñanza especial para los alumnos sordomudos y ciegos. El rectorado de Santiago, junto con las diputaciones gallegas, confeccionó las bases, que fueron aprobadas por R. O. del Ministerio de Instrucción Pública el 22 de noviembre de 1862. Posteriormente se redactó el Reglamento, aprobado igualmente por el Ministerio de Instrucción por R. O. del 25 de septiembre de 1863, y el 1 de junio de 1864 se inauguró el Colegio, bajo el patrocinio de la Universidad de Santiago, financiado por las cuatro diputaciones gallegas. El Colegio de Sordomudos y Ciegos de Santiago de Compostela representó "el primero de los que se ha fundado en España efecto de la Ley de instrucción pública del 9 de septiembre de 1857’’13, constituyendo, además, un colegio único en su tiempo en cuanto a organización y trayectoria pedagógica. El reconocimiento a su acertada organización se recoge tanto en publicaciones de la época como en otras mucho más recientes, como la siguiente de la Sociedad Pediátrica del año 2007:

\begin{abstract}
La Ley de Instrucción Pública de 1.857, conocida como Ley Moyano, en su artículo 108 establece que promoverá asimismo el Gobierno enseñanzas para los sordomudos y los ciegos, procurando que haya por lo menos una escuela en cada distrito universitario, y que en las públicas de niños se atienda, en cuanto sea posible, a la educación de aquellos desgraciados. En la práctica la aplicación de esta disposición fue desigual, existiendo centros con una pésima
\end{abstract}

\footnotetext{
${ }^{12}$ F. Fernández Villabrille, Estado actual y organización de la enseñanza de Sordomudos..., pág. 87.

${ }^{13}$ Manuel López Navalón, Memoria sobre el estado actual del Colegio de Sordo-Mudos y de Ciegos de Santiago. Mejoras de que es susceptible para su futuro progreso y prosperidad, Memoria, Santiago de Compostela, Imprenta Manuel Miras, 1867, pág. 3.
} 
organización mientras que otros, eran dignos de destacar como el de Galicia en Santiago que atendía a las cuatro provincias ${ }^{14}$.

En el eficaz funcionamiento del establecimiento no se puede obviar el respaldo de su primer director, el toledano Manuel López Navalón ${ }^{15}$. A juzgar por las palabras del dirigente republicano Emilio Castelar ${ }^{16}$, un sabio oculto y, según el profesor del Colegio Nacional de Sordomudos y Ciegos, Ricardo Gordo, el nombre de López Navalón, “con justos títulos puede figurar al lado de nombres destacados en la Educación como los de Hervás, Ballesteros, Nebreda, Ricart y Llorents"17. También, resultó indispensable el respaldo del por entonces Ministro de Fomento, Montero Ríos, que brindó su apoyo ante cualquier proyecto y reforma relacionada con el Colegio ${ }^{18}$. Asimismo, hay que resaltar el esfuerzo de las diputaciones provinciales, para el sostenimiento del establecimiento "Ninguna otra región de España puede vanagloriarse, como Galicia de haber sostenido un colegio de internado gratuito para sordomudos y ciegos de Galicia, desde el pasado siglo"19, e incluso el estímulo de la prensa regional y nacional, que ha contribuido a difundir tanto la problemática situación que tuvo que soportar el establecimiento como sus éxitos.

El Colegio de Sordomudos y Ciegos nació en una Galicia en la que la oligarquía, la burguesía y una iglesia fuertemente conservadora mantenían un férreo control de la sociedad; un contexto represivo en el que comenzaban a despuntar ciertos brotes republicanos, regionalistas y sindicalistas. En cuanto a la situación escolar, Galicia presentaba en las últimas décadas del siglo XIX un atraso general mucho

\footnotetext{
${ }^{14}$ José A. Díaz Huertas, Miguel A. Ruiz Díaz y Víctor Ruíz Díaz, Infancia y discapacidad, Madrid, Sociedad de Pediatría Social, 2007, pág. 11.

${ }^{15}$ Según la documentación que consta sobre López Navalón en el AHUS, en diciembre del año 1863 , es designado por la reina como director del Colegio, siendo nombrado en este cargo en abril de 1864 por el alcalde de la ciudad de Santiago. Esta documentación se encuentra en el Archivo histórico Universitario de Santiago de Compostela (en adelante solo AHUS), F. U: sordomudos y ciegos, caja 6870, exp. Manuel López Navalón.

16 "Don Manuel López Navalón, director del Colegio de Sordomudos y Ciegos de Santiago", El Mortero, 170 (6 de diciembre de 1897), pág. 1541.

${ }^{17}$ Ricardo GoRDo, "Don Manuel Navalón”, La Escuela Moderna, revista pedagógica y administrativa de primera y segunda enseñanza, 143 (Febrero1903), pág. 105.

${ }^{18}$ Para agilizar los trámites para la construcción de un nuevo edificio para el Colegio, resultó indispensable el ministro Eugenio Montero Ríos. Se pueden consultar los planos del nuevo Colegio en el AHUS, Edificios: Colegio de Sordomudos y Ciegos de Santiago de Compostela. Planos Colegio de Sordomudos y de Ciegos, 1909-1911, F. U. PLANOS, n. ${ }^{\circ} 84$.

19 "El Colegio Nacional de sordomudos y ciegos, un problema vivo de Galicia", El Sol (22 de julio de 1934), pág.10.
} 
mayor que el que sufría el resto de España ${ }^{20}$, aunque hay que puntualizar que no todas las provincias gallegas experimentaban dicho atraso por igual. Hacia los años cuarenta del siglo XIX, el provincialismo comenzó a germinar, como consecuencia de esta marginación. Un importante sector de la prensa gallega apoyó la difusión de este movimiento que luchaba por el reconocimiento de los valores de la tierra, sus costumbres, el arte, la historia y, posteriormente, por la reivindicación del uso de la propia lengua. Los aires regeneracionistas que se sentirán a finales del siglo XIX en todo el país ayudarían a fundamentar las críticas contra el régimen, además de servir a los galleguistas y ciertos grupos, como el de campesinos o emigrantes, a definir nuevos ideales.

Santiago de Compostela, sede del Colegio y centro urbano representativo del Reino durante siglos, padecía a mediados del siglo XIX un declive comercial y demográfico. Entre los años 1874 y 1900, la ciudad compostelana comenzó nuevamente a resurgir, por un lado, gracias al apoyo eclesiástico (con la recuperación de la tradición jacobea que fomentará las peregrinaciones al apóstol), y por otro, gracias al apoyo político, con el cual se revalorizó la condición de ciudad científica, con la consiguiente promoción de la enseñanza y la Universidad, donde se educarán (entre otros estudiantes) generaciones de jóvenes republicanos, galleguistas y progresistas ${ }^{21}$. El apoyo político se lo brindará el ministro Eugenio Montero Ríos, que, debido a los cargos gubernamentales que desempeñaba en Madrid, proporcionaba a la ciudad gallega concesiones civiles de relevancia.

El Colegio de Sordomudos y Ciegos, inaugurado el 1 de junio de 1864, se instaló en un primer momento en las dependencias de un antiguo convento cuya fundación se atribuye a San Domingo de Guzmán. Los alumnos de la escuela podrían ser internos o externos. Solo se admitían niños mayores de ocho años y menores de catorce. El número total de alumnos no podía superar los sesenta, y de estas plazas, cuarenta las ocupaban los internos elegidos por la Junta de Instrucción Pública de las cuatro provincias del distrito, y eran financiadas por los fondos de la escuela. Las veinte restantes se reservaban a los que las corporaciones o particulares quisiesen pensionar. Los sordomudos y ciegos matriculados en el Colegio de Santiago de Compostela recibían la enseñanza elemental y los conocimientos primarios básicos que les servirían como base a la futura enseñanza de ampliación e industrial. La duración de la enseñanza era de ocho años, cuatro

\footnotetext{
${ }^{20}$ En este sentido, consultar - entre otros- a Narciso de Gabriel Fernández, "Financiación de la escuela pública en la España del siglo XIX. El caso Gallego", Historia de la educación, revista interuniversitaria, 7 (1988), págs.165-167, Antón Costa Rico, Historia da educación e da cultura en Galicia. (Séculos IV-XX), Vigo, Xerais, 2004, pág. 733.

${ }^{21}$ Ramón Villares, "La ciudad de los 'Dos Apóstoles' (1875-1936)", en Ermelindo Portela Silva (coord.), Historia de la ciudad de Santiago de Compostela, Santiago de Compostela, Universidad de Santiago de Compostela, 2003, págs. 465-480.
} 
de los cuales se dedicaban a la instrucción elemental básica, y los otros cuatro, a la enseñanza de ampliación o industrial ${ }^{22}$.

El establecimiento era una prolongación del hospicio de pobres reservado a los naturales de Santiago. El departamento de niños ocupaba parte del ala derecha del piso principal. Se dividía en un lavatorio para el aseo y limpieza y dos clases bastante espaciosas. Además había un extenso salón dormitorio con treinta camas, a cuyos extremos se encontraba la habitación de los ayudantes, que vigilaban a los internos a todas horas, y una enfermería para casos leves. El departamento de niñas se hallaba situado en el ala izquierda del piso segundo, y se dividía también en un lavatorio y dos clases, un dormitorio y dos habitaciones más para cuando las necesidades del servicio lo exigiesen. Las habitaciones eran muy claras y suficientemente ventiladas, con exposición sudoeste ${ }^{23}$. No obstante, el Colegio, dependiente de la Universidad y relegado a ese ángulo del orfanato -según el testimonio de su primer director-, nunca había podido sentirse como "tal Colegio".

En 1868 se llevaron a cabo ciertas reformas que servirían para dar mayor amplitud a las clases; además, se había construido una entrada independiente al Colegio (de la que hasta entonces carecía). En la planta baja del edificio se había construido un salón grande, donde se impartían la clase elemental de ciegos y la de música. Pero esas mejoras eran insuficientes: las habitaciones eran pocas, y el Colegio no contaba con un espacio exterior al aire libre, como un patio o jardín, que según el director favorecería el desarrollo de los alumnos.

Por otra parte, el régimen alimenticio no era saludable, y el menú no variaba. En palabras del director del Colegio, "Es mezquino por su cantidad, nada nutritivo por su calidad, y peor condimentado" 24 . La alimentación era básicamente vegetal. Se les daba a los alumnos por la mañana una taza de sopas sin pan. Al mediodía, una taza de caldo con berza o patata, o berza y habichuela, y una ración de pan. En la cena se repartía a los niños lo mismo que en la comida. Las niñas hospicianas eran las encargadas de preparar los alimentos. La falta de sal, la poca cocción o la abundancia de grasa causaban a menudo el rechazo de la comida. Asimismo, la mala calidad de productos, como el pan, ocasionaba denuncias como la que sigue:

\footnotetext{
${ }^{22}$ Colegio de Sordomudos y Ciegos de Santiago de Compostela, Bases y Reglamento para el establecimiento y régimen del Colegio de Sordomudos y de Ciegos del distrito universitario de Santiago, Santiago de Compostela, Imprenta Manuel Mirás, 1863, págs. 10-11.

${ }_{23}$ M. López Navalón, Memoria sobre el estado actual del Colegio de Sordo-Mudos y de Ciegos de Santiago. Mejoras de que es susceptible..., pág. 21.

${ }^{24}$ Manuel López Navalón, Memoria sobre el estado del Colegio de Sordo-Mudos y de Ciegos de Santiago y reformas que deben plantearse para su mejor organización, Santiago de Compostela, Imprenta Manuel Miras, 1870, pág. 32.
} 


\begin{abstract}
No hace mucho tiempo que se formó expediente judicial a un contratista porque analizado el pan a causa de quejas continuas, se vio, y en las declaraciones consta, que era amasado con las barreduras de la tahona y los desperdicios de la masa que cuidadosamente se recogían para este objeto ${ }^{25}$.
\end{abstract}

En cuanto a la ropa y al calzado, durante los primeros años de funcionamiento del Colegio los niños solo tenían un traje, que usaban tanto dentro del establecimiento como en los paseos y los actos públicos. Posteriormente se consiguió, gracias a los fondos del Colegio, que los alumnos tuviesen un traje nuevo para las presentaciones públicas, además de blusas para las clases. Con el calzado sucedía lo mismo: los niños iban descalzos la mayor parte del año.

Esta precaria situación que atravesaba el Colegio, y que afectaba por igual a los establecimientos de enseñanza de sordomudos y ciegos creados según las disposiciones de la Ley del 1857, no impediría el progreso de los alumnos. El dominio de las asignaturas adquirido por los ciegos y sordomudos del Colegio de Santiago se demostraba en distintos eventos públicos. El director, López Navalón, asistía a concursos y exposiciones acompañado de sus alumnos. El Colegio obtuvo la medalla de oro con diploma en el concurso celebrado en Santiago en el año 1875 por iniciativa de la Sociedad Económica; dos años después, en 1877, en la exposición que tuvo lugar en la ciudad de Lugo, se le adjudicó otra medalla de oro, y con el paso del tiempo y en repetidas ocasiones el Colegio seguiría obteniendo premios, dando fe de los logros de los alumnos y del excelente trabajo de su director. Pero no solo son destacables sus méritos grupales honoríficos, sino que la labor de enseñanza fue crucial en la trayectoria vital de algunos alumnos.

Desfilaron por sus aulas ilustres músicos ciegos que se ganaron la vida tocando (artistas de renombre, como Alfonso Couto y profesores destacados como Enrique Rodeiro o Francisco Méndez, que estuvo al frente de la escuela municipal de ciegos de Vigo). Incluso algunos intelectuales han conseguido con sus obras traspasar las fronteras españolas, como el sordomudo José María Acuña, célebre escultor calificado como el último de los grandes clásicos del arte gallego, que fue además el primer docente sordo de Galicia ${ }^{26}$, y Juan Lorenzo González ${ }^{27}$. Este alumno, que destacó entre los demás nada más ser inaugurado el Colegio gallego, llegaría a convertirse en el primer profesor de ciegos de Argentina.

\footnotetext{
${ }^{25}$ M. López Navalón, Memoria sobre el estado del Colegio de Sordo-Mudos y de Ciegos de Santiago y reformas que deben plantearse..., pág. 33.

${ }^{26}$ Javier Mosquera, "José María Acuña hizo de su arte su voz", Faro de Vigo (12 de julio de 2012) [en línea], disponible en <http://www.farodevigo.es/> [consulta : 07/08/2014].

27 "Hombres de mérito, el director de nuestro Colegio Regional de sordomudos y ciegos", El diario de Pontevedra (29 de noviembre de 1927), pág. 1.
} 
La información sobre Juan Lorenzo González es prácticamente inexistente. El historiador Alberto Vilanova, uno de los investigadores que más ha trabajado por la recuperación del pasado de Galicia, dedica un breve apartado al maestro ciego $^{28}$ en su obra Los gallegos en la Argentina. Su estudio, que se remonta a los años cincuenta del siglo XX, reúne datos significativos sobre Juan Lorenzo. Sin embargo, el historiador se lamenta de la carencia de documentación sobre su vida y obra. Es más, el uso de su nombre es ambiguo, ya que en ocasiones se refieren a su persona nombrándole como José o Manuel Lorenzo y González ${ }^{29}$, en lugar de Juan. El mismo Alberto Vilanova (1966) titula el apartado que le dedica al maestro en la obra anteriormente citada, José Lorenzo González, el primer profesor de la Escuela de ciegos, aunque reconoce que indistintamente se le nombra como José, Juan o Manuel. En el Boletín de la Academia Nacional de Medicina de Buenos Aires, volumen 71, del año 1993, también se cita con el nombre de José. En otros estudios aparece con el nombre de Juan, nombre con el que se le cita en El Simposio internacional de lectura y escritura y nuevos desafios (2002).

El desconocimiento de Juan Lorenzo González se hace patente en lo expuesto. No obstante, a falta de cualquier otra pincelada biográfica, la indagación hecha sobre su persona parece confirmar que el nombre por el que se conocía a este relevante pedagogo español en el colegio donde estudió y ejerció como profesor era Juan, no Manuel ni José, y que procedía de la misma ciudad en la que realizó sus estudios: la ciudad gallega de Santiago de Compostela.

La probabilidad de que Juan Lorenzo González hubiera estudiado en el colegio de esta ciudad era muy alta, ya que en principio existen pocas opciones de que hubiese podido cursar la instrucción elemental en otro colegio. Los establecimientos de enseñanza especial que se fueron inaugurando en las distintas capitales españolas durante la segunda mitad del siglo XIX eran escasísimos ${ }^{30}$. Pero aparte de la alta probabilidad, se dispone de datos significativos que confirman que Juan Lorenzo González estudió en el Colegio de Santiago de Compostela: su nombre figura entre los alumnos externos del Colegio, y, además, como natural de esta ciudad gallega ${ }^{31}$.

En la hoja de observaciones de la primera Memoria del Colegio del año 1867 aparece su nombre como uno de los ciegos más notables: "De los ciegos son los más notables Celestino Martínez Bentrón por la Coruña, José López por Lugo y

\footnotetext{
${ }^{28}$ Hay que puntualizar que Juan Lorenzo González no era completamente ciego.

${ }^{29}$ Cabe destacar que en ocasiones se citan los apellidos, Lorenzo González, con una "y" en medio como separación, lo que era una costumbre muy generalizada a finales del siglo XIX.

${ }^{30}$ Pedro Molina Martín, "Los Institutos de sordomudos y ciegos", La Escuela Moderna, revista pedagógica hispano-americana, 111 (junio de 1900), pág. 455.

${ }^{31}$ M. López Navalón, Memoria sobre el estado actual del Colegio de Sordo-Mudos y de Ciegos de Santiago. Mejoras de que es susceptible..., pág. 35.
} 
Juan Lorenzo González, alumno externo"32. En este tiempo, apenas inaugurado el Colegio, los alumnos ciegos leían con soltura y facilidad en los dos sistemas de relieve convencionales de puntos y caracteres usuales españoles. También escribían en dos sistemas distintos: el convencional de puntos formando relieve y en aparato mecánico. En aritmética, los alumnos más aventajados dominaban teórica y prácticamente la materia. Además, poseían nociones elementales de geografía, astronómica, física y política, geometría, religión, historia sagrada y gramática ${ }^{33}$.

El propio director fue quien, una vez inaugurado el Colegio, consiguió el material necesario para sus alumnos. Dicho material no solo servía para la clase de sus alumnos, sino que se presentó en diferentes exposiciones (entre ellas, la exposición Universal de París del año $1867^{34}$ ). El ingenio y el conocimiento pedagógico que demostraba Manuel López Navalón, en su faceta de inventor y creador de aparatos de finalidad didáctica para la enseñanza especial, también lo llevó a introducir alguna innovación respecto al braille. Cabe destacar que los signos alfabéticos usados en el Colegio de Santiago no eran iguales a los del Colegio Nacional de Madrid ${ }^{35}$; la numeración tampoco era la misma ${ }^{36}$.

Asimismo, López Navalón modificó el sistema de notación musical empleada por los ciegos en Madrid. El método seguido por el Colegio madrileño no le parecía sencillo: existía demasiada confusión en los signos que expresaban la nota y su valor, y el tacto del ciego, aunque delicado, no podía apreciarlos con la rapidez

\footnotetext{
${ }^{32}$ M. López Navalón, Memoria sobre el estado actual del Colegio de Sordo-Mudos y de Ciegos de Santiago. Mejoras de que es susceptible..., pág. 37.

${ }_{33}$ M. López Navalón, Memoria sobre el estado actual del Colegio de Sordo-Mudos y de Ciegos de Santiago. Mejoras de que es susceptible..., pág. 24.

${ }^{34}$ El Colegio de Santiago de Compostela, recientemente inaugurado, no se encontraba en condiciones de enviar a ningún representante a París, pero López Navalón remitió dos cajas para el estudio de la geometría (AHUS, F.U: sordomudos y ciegos, caja 6870, exp. Manuel López Navalón).

${ }^{35}$ El Colegio de Sordomudos y Ciegos de Madrid constituía una escuela completa con profesorado para las enseñanzas generales, especiales y profesionales, con instalaciones adecuadas, material pedagógico especial y capacidad para un número de alumnos significativo. Por todo ello no solo servirá de modelo a los demás establecimientos de enseñanza, sino que asumirá prácticamente todas las iniciativas e incidencias de la educación especial española durante las primeras décadas del siglo XX (Mariano HerRaiz Gascueña, Aproximación a la educación especial española del primer tercio del siglo XX, Murcia, Compobell, 1995, pág. 17).

${ }^{36}$ Como comenta el profesor del Colegio Nacional madrileño, Pedro Molina, "Los signos innovados por el Sr. Navalón son mixtos, en cuanto que á su determinación ó fijeza ha presidido el deseo de que por el número de sus puntos constitutivos recuerden el número de unidades representadas por la cifra arábiga; y cuando esto no ha sido posible, supuesto que el máximum de elementos que entran en la combinación es seis, se intentó que los signos arbitrarios asemejen, en cuanto cabe, la figura gráfica de los ordinarios" (Pedro Molina MARTín, "Procedimiento para la enseñanza de la aritmética de los ciegos", La Escuela Moderna, revista pedagógica hispano-americana, 30 de setiembre de 1983, pág. 192).
} 
requerida. Por ello trató de simplificar el sistema musical ${ }^{37}$. Por otra parte, el método de piano que se utilizaba en el Colegio de Madrid, en opinión de López Navalón, también resultaba complicado para los alumnos ciegos: se escribía la parte correspondiente a cada mano en una misma línea, entremezclando la mano izquierda con la derecha (lo que producía confusión). Este método fue modificado presentando las lecciones a los alumnos de igual forma que a los videntes: el estudio de la mano derecha en la parte superior, y el de la izquierda, en la inferior ${ }^{38}$.

Los métodos didácticos utilizados por el director resultaban eficaces. En el año 1870, el progreso de los alumnos era admirable. En este año, en la lista de alumnos externos aparece de nuevo el nombre de Juan Lorenzo González. En el apartado de observaciones consta que desempeñaba el cargo de auxiliar de la clase de $\operatorname{ciegos}^{39}$.

Habían transcurrido seis años desde el inicio de las clases, y los ciegos que habían ingresado en el año de inauguración del Colegio ya habían terminado la enseñanza elemental, y después de haber adquirido las nociones básicas, dedicaban la mayor parte del tiempo a la enseñanza musical ${ }^{40}$. En cualquier caso, los alumnos del colegio aumentaban, y las horas de clase no eran suficientes para atenderlos a todos. Si bien el personal del Colegio reunía en sus inicios a un maestro director, un segundo profesor, dos ayudantes, una maestra de labores, una ayudante y un conserje portero, en el año 1870 tendrían que sumarse a este personal dos ayudantes, un auxiliar y un profesor de música. Los procedimientos especiales utilizados, tanto en la enseñanza de ciegos como la de sordomudos, requerían la división de los alumnos en diferentes secciones, llegando a tener que recurrir a la enseñanza individual:

El aumento de personal para la enseñanza debe hacerse; pues dos profesores para cuarenta alumnos, treinta internos y diez externos, de dos desgracias dos sexos y dos enseñanzas distintas, divididos y subdivididos en secciones que cada una necesita las seis horas diarias de instrucción no bastan por mucho que se desvelen y por mucho que sea su celo y buenos deseos, para enseñar a todos cual se exige y cual su desgracia respectiva lo reclama ${ }^{41}$.

\footnotetext{
${ }^{37}$ Ricardo GoRdo, "Don Manuel...", pág 105.

${ }_{38}$ M. López Navalón, Memoria sobre el estado del Colegio de Sordo-Mudos y de Ciegos de Santiago y reformas que deben plantearse..., pág. 22.

${ }^{39}$ M. López Navalón, Memoria sobre el estado del Colegio de Sordo-Mudos y de Ciegos de Santiago y reformas que deben plantearse..., pág.48.

${ }^{40}$ M. López Navalón, Memoria sobre el estado del Colegio de Sordo-Mudos y de Ciegos de Santiago y reformas que deben plantearse..., pág. 24.

${ }^{41}$ Manuel López Navalón, Memoria sobre el estado del Colegio de Sordo-Mudos y de Ciegos de Santiago y reformas que deben plantearse..., pág. 38 .
} 
Las exigencias didácticas obligaron a López Navalón a solicitar a las autoridades pertinentes la creación de una plaza de profesor auxiliar, proponiendo a uno de sus alumnos, Juan Lorenzo González, para ocupar este cargo ${ }^{42}$. El director lo consideraba un discípulo aventajado, capacitado para ayudarle a impartir la clase de los ciegos. Para llevar a cabo esta reforma, entre otras de las planteadas por el director en beneficio del establecimiento, se solicitó a cada una de las cuatro provincias gallegas (que eran las que financiaban el Colegio) que aumentasen anualmente una cantidad. A esto solo se prestaron las de Lugo y A Coruña. Al no acceder las otras dos provincias, no se podía llevar a cabo la reforma pretendida en su totalidad, por más que López Navalón solicitó y suplicó -según la solicitud expedida al respecto- que se hiciera la reforma parcial y que se concediera la susodicha plaza al aspirante Juan Lorenzo González, en atención a sus méritos y servicios. En realidad, esta labor ya la estaba desempeñando Juan Lorenzo desde el 1 enero del año 1868, con notable acierto (aunque gratuitamente). Si bien el 1 de junio de ese mismo año recibió el encargo de transportar la escritura musical del sistema ordinario al especial (es decir, en relieve para ciegos), tan solo fue retribuido por esta tarea con ocho escudos mensuales ${ }^{43}$.

Pero aparte de la experiencia en estas funciones didácticas, Lorenzo González se encontraba preparado para ocupar la plaza, ya que había cursado y aprobado dos años en la Escuela Normal de la ciudad para obtener el título de maestro elemental. La causa de que no ejerciese el magisterio se encontraba, según apunta López Navalón, en sus limitaciones físicas. Cabe destacar que no era completamente ciego. El plan de estudios vigente de las Escuelas Normales en la década de los años sesenta del siglo XIX se organizaba según las disposiciones de la Ley Moyano. El segundo maestro y secretario de la Escuela Normal Superior de Santiago, Don Juan Codina Iglesias, certificó las asignaturas cursadas y aprobadas por Juan Lorenzo González durante el período de $1865-1866^{44}$. Las calificaciones recibidas en dichas asignaturas variaban entre el mediano y aprobado.

Entre los años 1865-1866, Juan Lorenzo González cursó y aprobó las siguientes asignaturas:

- Doctrina cristiana y nociones de historia sagrada.

- Lengua castellana, ejercicios de análisis, composición y ortografía.

- Aritmética.

\footnotetext{
${ }^{42}$ AHUS, Fondo Universitario: Escuelas Especiales/sordomudos y ciegos/ General, caja: 6870, Exp de Lorenzo y Gonzalez, Juan, pág. 5.

${ }^{43}$ AHUS, caja: $6.870 \ldots$, pág 1.

${ }^{44}$ AHUS, caja: $6.870 \ldots$, pág. 3.
} 
En los exámenes ordinarios del curso 1868-1869, las asignaturas aprobadas fueron las siguientes:

- Doctrina cristiana y nociones de historia sagrada.

- Lengua castellana, ejercicios de análisis, composición y ortografía.

- Elementos de geografía y nociones de historia de España.

- Nociones de geometría, dibujo lineal y agrimensura.

- Nociones de agricultura.

- Principios de educación y métodos de enseñanza.

Las huellas del maestro ciego se pierden fuera del ámbito académico. El motivo que le impulsó a abandonar su ciudad gallega natal y realizar el largo viaje hacia América no está del todo claro; lo cierto es que fuera cual fuese su motivación, la misión educativa que desempeñó a su llegada a Buenos Aires constituyó una gran obra pedagógica. Si bien en el año 1857 la Sociedad Filantrópica Regeneración fundó en Argentina la primera escuela para sordos con la llegada del pedagogo español, se iniciaba un nuevo capítulo de la educación especial de este país del sur de América. El siguiente párrafo, recogido del Simposio Internacional ${ }^{45}$ celebrado en Argentina sobre la investigación del braille, testimonia lo dicho: "La enseñanza sistemática del braille en nuestro país se remonta al año 1887, cuando la Sociedad de Beneficencia contrató al maestro ciego Juan Lorenzo y González para enseñar en el asilo".

El PRIMER INTENTO DE ENSEÑAnZA Sistematizada DEL CIEGo en Argentina: JUAN LORENZO GONZÁLEZ, PRIMER PROFESOR DE CIEGOS

El sistema braille se dio a conocer en Argentina en el Asilo de Huérfanos de Buenos Aires. Este establecimiento, según consta en el tomo I de las Instituciones de la Sociedad de Beneficencia y asistencia social de Buenos Aires ${ }^{46}$, fue considerado "una institución ejemplar por su organización y funcionamiento, y por la novedosa inclusión de una escuela para ciegos, que funcionó dentro del Asilo, entre 1887 y 1909”.

\footnotetext{
${ }^{45}$ María Alejandra Grzona, "Investigación del sistema braille”, en El Simposio Internacional de lectura y escritura y nuevos desafios (Argentina, 4-6 de abril del 2002), Cuyo, Universidad Nacional de Cuyo, pág. 3.

${ }^{46}$ Instituciones de la Sociedad de Beneficencia y Asistencia Social (1823-1952), Documentos escritos, tomo I, Buenos Aires, 1999, pág. 43.
} 
Los orígenes de esta escuela se remontan al año 1886, cuando la epidemia de viruela que se desató en Argentina dejó un cuantioso número de fallecidos y graves secuelas entre los supervivientes. Varios menores, que se habían quedado ciegos a consecuencia de la enfermedad, fueron ingresados en la Casa de Expósitos. Posteriormente fueron trasladados a un pabellón del Asilo de Huérfanos para recibir una educación adecuada a sus posibilidades. El director de esta institución, el presbítero Bernabé Pedernera, se encargó de hacer efectiva dicha labor redentora inmediatamente. Para ello precisaba la colaboración y el asesoramiento de un experto en la materia, y el elegido fue el sacerdote italiano Escipión Mercati, versado en estos conocimientos al haber seguido cursos de tiflología en Italia. No obstante, el plan de enseñanza no llegaría a concretarse, debido al desacuerdo entre los propósitos del sacerdote y el reglamento general vigente del Asilo.

A principios de marzo de 1887, la presidenta de la Sociedad de Beneficencia, Luisa Muñoz de Cantilo, propuso para dicha tarea a Juan Lorenzo y González, que, procedente de Santiago de Compostela, acababa de llegar a Buenos Aires. El propósito que había llevado al maestro ciego a realizar tan largo viaje fue el de conseguir la financiación suficiente para inaugurar un colegio para ciegos en esta ciudad ${ }^{47}$, aunque otra de las posibles hipótesis que se baraja es que ya venía contratado desde España para ejercer la enseñanza en el Asilo de Huérfanos.

El 1 de marzo de 1887, la dirigente de la Sociedad de Beneficencia de Buenos Aires, la Sra. Luisa Muñoz de Cantilo de Villate, dirigió al Ministro del Interior el siguiente escrito, en el que se proponía a Juan Lorenzo González para desempeñar la plaza vacante de profesor en el Asilo de Huérfanos:

Tengo el agrado de dirigirme a V. E. para comunicarle que, estando vacante la plaza de profesor de ciegos en el Asilo de Huérfanos, la Corporación que presido me encarga proponer al Señor Ministro el nombramiento del Sr. José Lorenzo González para desempeñar este puesto. Esperando que esta elección merecerá la aprobación de $V$. E. reitero la seguridad de mi distinguida consideración ${ }^{48}$.

Esta propuesta fue aceptada rápidamente, y el 28 de mayo de 1887 el Sr. González fue nombrado profesor de la primera escuela de ciegos Unos meses

\footnotetext{
47 Julia E. CASAÑAS DE Rico, Los ciegos: educación, instrucción, relaciones humanas, trabajo, Buenos Aires, Ediciones Crisol, 1977, pág. 15.

48 Alberto Vilanova Rodriguez, Los gallegos en la Argentina, tomo II, Buenos Aires, Ediciones Galicia, 1966, pág. 957.
} 
después inició su tarea enseñando a los niños ciegos en el Asilo de Huérfanos ${ }^{49}$. La atención institucional y profesional de los ciegos en Argentina se inició en este momento. El abandono social había sido la tónica general que hasta entonces acompañaba a estos discapacitados. Su único medio de supervivencia era la asistencia caritativa o la mendicidad.

En la escuela, Juan Lorenzo González enseñaba a sus alumnos a leer y escribir, además de algunas nociones de aritmética y manualidades para completar la instrucción elemental de los alumnos. Las clases, en un principio, se destinaban solamente a los varones, pero unos meses después se estableció un curso para mujeres. Cabe señalar que en las clases de este maestro se utilizó el primer aparato que sirvió para la enseñanza del Braille en el país ${ }^{50}$. Este aparato fue donado años más tarde por su hijo, Victoriano Lorenzo, con motivo del acto inauguración de la imprenta de la Biblioteca Argentina para ciegos.

Si bien apenas existe información sobre el desempeño del magisterio de Juan Lorenzo, según la información recogida por Alberto Vilanova (1966) del testimonio de un antiguo alumno, ha quedado constancia que el trato con sus discípulos era paciente y cariñoso. El interés que mostraba en el aprendizaje de sus alumnos se evidencia en su preocupación por renovar el material de la escuela, solicitando que le fuesen enviados desde París libros en relieve de historia natural, que maravillaban a los alumnos. Asimismo, en un acta que corresponde al tomo 2 del Asilo de Huérfanos (1885-1887) de la Sociedad de Beneficencia, en sus páginas 3 y 4 se alaba la labor didáctica de este maestro:

\begin{abstract}
Examináronse en este día en lectura, escritura y aritmética y geografia 10 alumnos ciegos, de cuya enseñanza está encargado el Sr. Juan Lorenzo y González [Como se ve, el maestro recibe indistintamente tanto los nombres de Juan como José, en actos y documentos oficiales], habiendo quedado la concurrencia agradablemente impresionada por el adelanto de algunos de aquellos infelices que según se vé no estarán en lo sucesivo condenados a vivir en la ignorancia, sino que podrán ver la luz de la ciencia, ya que nó la del sol ${ }^{51}$.
\end{abstract}

La labor de Juan Lorenzo González al frente de la escuela se prolongó hasta 1892, año en el que fue reemplazado por el maestro italiano Francisco Gatti, un educador napolitano que había llegado a Buenos Aires dos años antes. Se

\footnotetext{
49 Julia E. CASAÑAS DE RICO, Los ciegos: educación, instrucción..., pág. 15

${ }^{50}$ A. Prignano, Una Historia de Relieve. Biblioteca ..., pág. 34.

${ }^{51}$ A. Vilanova Rodriguez, Los gallegos en la..., pág. 958.
} 


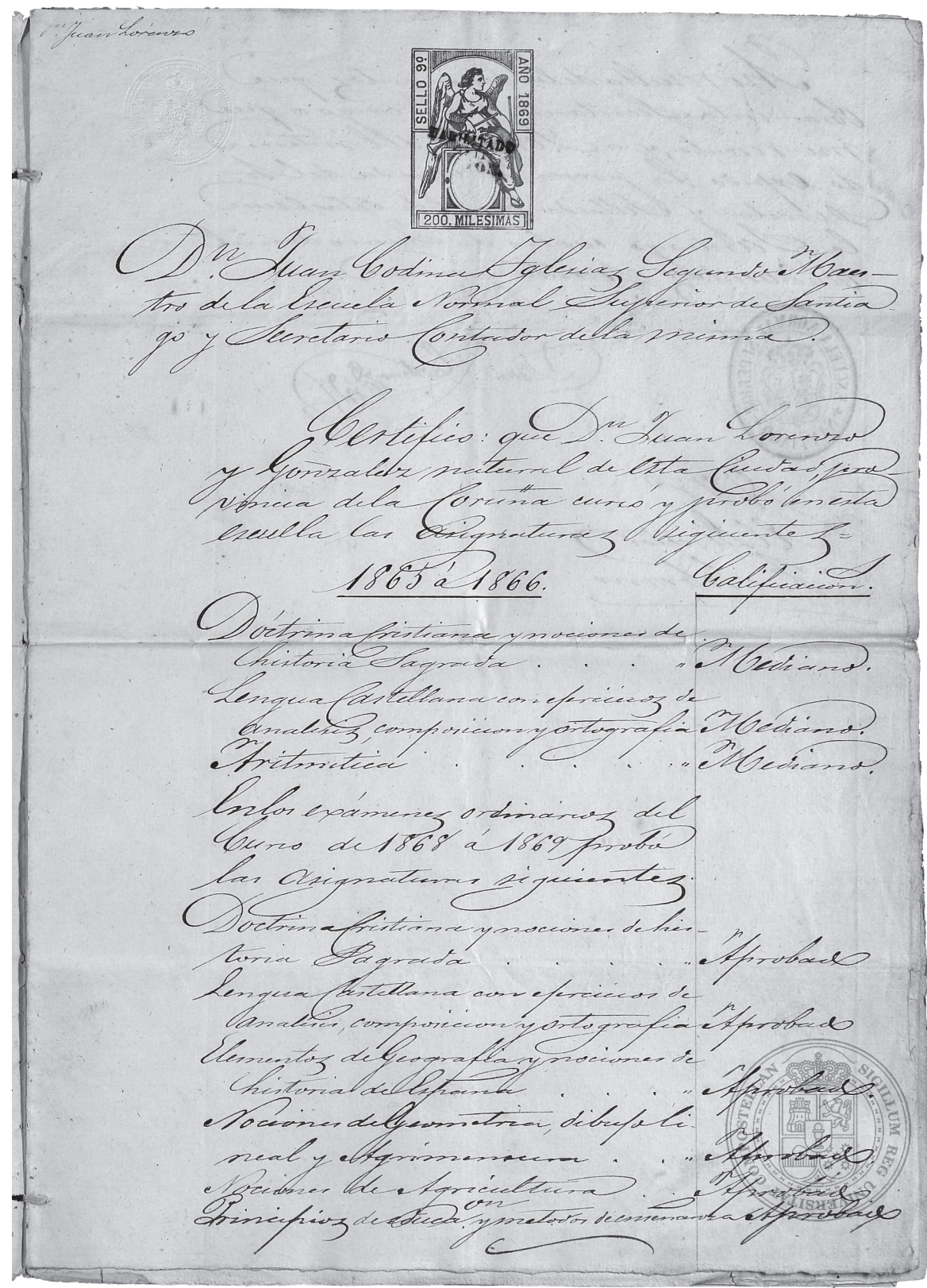

Figura 1. Calificaciones recibidas por Juan Lorenzo González, en las asignaturas cursadas durante los años 1865-1869.

(AHUS, Fondo Universitario: Escuelas Especiales/sordomudos y ciegos/General, caja: 6.870, Exp. de Lorenzo y Gonzalez, Juan, pág. 3) 
desconoce el motivo de dicha sustitución: quizá se debió a que la plaza se encontraba vacante en esos momentos. Ángel Prignano (2004) sostiene que la posible causa del relevo del maestro gallego por el italiano fue que Juan Lorenzo no poseía los suficientes conocimientos musicales. Esta carencia era imperdonable para un maestro de la época, en la que a los ciegos se les orientaba principalmente hacia la música, considerada la ocupación más apropiada para un invidente: "La música sin excepción alguna, debe entrar en el plan de enseñanza de todos los ciegos, pues este arte es para ellos el medio más socorrido de subsistencia. Todos los ciegos deben aprender a tocar algún instrumento [...]"52.

Pese a la labor desempeñada en la escuela de ciegos, el nombre de Juan Lorenzo González apenas se menciona: "si bien se habla de la creación de la escuela de ciegos, se omite inexplicablemente el nombre de su benemérito primer profesor" ${ }^{53}$. En un artículo de la revista argentina ${ }^{54}$ Caras y caretas del año 1901, que hace referencia a la enseñanza de los ciegos en Buenos Aires, se relata detalladamente la vida y obra del profesor italiano Gatti, pero se ignora la presencia del maestro español.

En el año 1903 se publicó en Argentina una memoria sobre enseñanza de ciegos y proyecto de organización de un primer Instituto Nacional. En dicha memoria, que recogía las iniciativas que habían surgido para proporcionar instrucción a los ciegos en el país, se hace mención a la escuela de ciegos del Asilo de Huérfanos, pero no se cita al maestro gallego:

\begin{abstract}
Apareció primero una pequeña sección anexa al Asilo de huérfanos que ha venido funcionando en condiciones dificiles, porque no solo ha tenido que luchar con la falta de local aparente y de los útiles indispensables sino también del personal suficiente para impartir con éxito la enseñanza. Esta escuela ha sido frecuentada anualmente por 25 ó 30 alumnos de los dos departamentos; pero en la actualidad su número es más reducido. La otra escuela existente es de propiedad particular y fue fundada por el señor Gatti (ciego). Goza de una subvención nacional y solo es frecuentada por ocho alumnos ${ }^{55}$.
\end{abstract}

\footnotetext{
52 Juan Manuel Ballesteros, Curso elemental de instrucción de ciegos y de sordomudos, Madrid, Imprenta del Colegio Nacional de Sordomudos, 1862, pág 230.

${ }_{53}$ A. Vilanova Rodriguez, Los gallegos en la ..., pág. 957.

54 "La enseñanza de un ciego por otro ciego", Caras y caretas (15 de junio del año 1901), págs. 33-34.

${ }^{55}$ Ulises Codino, Memoria sobre enseñanza de ciegos y proyecto de organización de un primer Instituto Nacional presentados al Ministerio de Instrucción Pública, Buenos Aires, Imprenta Boullosa, 1903, pág. 16.
} 
Si bien es cierto que el maestro italiano Francisco Gatti fue quien impulsó definitivamente a la enseñanza de los ciegos en Argentina, propiciando la creación del instituto para ciegos de ambos sexos, como afirma Prignano (2004): “...A Don Juan Lorenzo y González nadie le podrá quitar el alto honor de ser el primer maestro para ciegos de la Argentina" $"$.

\section{CONCLUSIONES}

De acuerdo con lo expuesto en este trabajo, no cabe duda del desconocimiento que existe sobre Juan Lorenzo González; no obstante, se constatan mediante la documentación que aquí se presenta ciertos datos y se desvela información significativa que permite esclarecer la confusa biografía del maestro ciego.

Ante la documentación examinada, podemos demostrar que Juan Lorenzo González era gallego, natural de la ciudad de Santiago de Compostela, y que pasó parte de su vida en Galicia. Fue un alumno destacado, y también desempeñó las funciones de profesor en el Colegio de Sordomudos y Ciegos de Santiago de Compostela en los primeros años de su inauguración.

En cuanto a su labor pedagógica, se constata que la contribución de Juan Lorenzo González en los inicios de la enseñanza especial de las personas con discapacidad visual ha sido relevante. Las limitaciones visuales que él mismo padecía no le impidieron trasladarse a Argentina, a principios del año 1887, con su título de maestro, y comenzar a educar a un grupo de niños ciegos del Asilo de Huérfanos de Buenos Aires. No fue una misión educativa nada fácil en los primeros tiempos en que se iniciaban estas enseñanzas, debido a la escasez de material y a la precaria situación económica que soportaban este tipo de escuelas e instituciones. Pese a ello, Juan Lorenzo González desempeñó sus funciones hasta el año 1892, considerándose esta labor como el primer intento de enseñanza sistematizada del ciego en Argentina.

\section{BIBLIOGRAFÍA}

Aguado Díaz, Antonio, Historia de las deficiencias, Madrid, Once, 1995.

Ballesteros, Juan Manuel y Fernández Villabrille, Francisco, Curso elemental de instrucción de ciegos y de sordomudos, Madrid, Imprenta del Colegio Nacional de Sordomudos, 1862

Burgos Bordonau, Esther, Historia de la enseñanza musical para ciegos en España, 1830-1938, Madrid, ONCE, 2004.

Casañas de Rico Contreras, Julia Esther, Los ciegos: educación, instrucción, relaciones humanas, trabajo, Buenos Aires, Ediciones Crisol, 1977.

\footnotetext{
${ }^{56}$ A. Prignano, Una Historia de Relieve. Biblioteca..., pág. 10.
} 
Codino, Ulises, Memoria sobre enseñanza de ciegos y proyecto de organización de un primer Instituto Nacional, Buenos Aires, Imprenta Boullosa, 1903.

Colegio de Sordomudos y Ciegos de Santiago de Compostela, Bases y Reglamento para el establecimiento y régimen del Colegio de Sordomudos y de Ciegos del distrito universitario de Santiago, Santiago de Compostela, Imprenta Manuel Mirás, 1863.

Costa Rico, Antón, Historia da educación e da cultura en Galicia. (Séculos IV-XX), Vigo, Xerais, 2004.

Fernández Villabrille, Francisco, Estado actual y organización de la enseñanza de Sordomudos y ciegos. Memoria dirigida al Ministro de Fomento, Madrid, Imprenta del Colegio de Sordomudos, 1862.

Gabriel Fernández, Narciso de, "Financiación de la escuela pública en la España del siglo XIX. El caso Gallego", Historia de la educación, revista interuniversitaria, 7 (1988), págs. 163-178.

Garvía Soto, Roberto, La Organización Nacional de Ciegos: un estudio institucional, Madrid, Centro de Estudios Avanzados en Ciencias Sociales, 1992.

Gordo, Ricardo, "Don Manuel Navalón”, La Escuela moderna, revista pedagógica y administrativa de primera y segunda enseñanza, 143 (febrero de 1903), págs. 105-114.

Grzona María Alejandra, "Investigación del sistema Braille”, en El Simposio Internacional de lectura y escritura y nuevos desafios (Argentina, 4-6 de abril del 2002), Cuyo, Universidad Nacional de Cuyo, págs. 1-5.

Instituciones de la Sociedad de Beneficiencia y Asistencia Social (1823-1952), Documentos escritos, tomo I, Buenos Aires, 1999.

López Alonso, Carmen, La pobreza en la España medieval, Madrid, Ministerio de Trabajo y Seguridad Social, 1986.

López Navalón, Manuel, Memoria sobre el estado actual del Colegio de Sordo-Mudos y de Ciegos de Santiago. Mejoras de que es susceptible para su futuro progreso y prosperidad, Santiago de Compostela, Imprenta Manuel Miras, 1867.

López Navalón, Manuel, Memoria sobre el estado del Colegio de Sordo-Mudos y de Ciegos de Santiago y reformas que deben plantearse para su mejor organización, Santiago de Compostela, Imprenta Manuel Miras, 1870.

Mackenzie, Clutha, La escritura Braille en el mundo, París, Unesco, 1954.

Marco, Aurora y Porto Ucha, Ángel Serafín, A Escola Normal de Santiago de Compostela, Santiago de Compostela, Servicio de Publicacións da Universidade de Santiago de Compostela, 2000.

Molina Martín Pedro, "Procedimiento para la enseñanza de la aritmética de los ciegos", $L a$ Escuela moderna, revista pedagógica hispano-americana, 30 (septiembre de 1893), págs. 184-195.

Molina Martín, Pedro, "Los Institutos de sordomudos y ciegos", La Escuela Moderna, revista pedagógica hispano-americana, 111 (junio de 1900), págs. 438-454.

Montoro Martínez, Jesús, Los ciegos en la historia, vol. 5, Madrid, ONCE, 1995.

Mosquera, Javier, “José María Acuña hizo de su arte su voz”, Faro de Vigo (12de julio de 2012) [en línea], disponible en $<$ http://www.farodevigo.es/> [Consulta: 07/08/2014]. 
TRAS LA HUELLA DEL DESCONOCIDO MAESTRO CIEGO DEL COLEGIO DE SORDOMUDOS Y CIEGOS DE SANTIAGO: JUAN LORENZO GONZÁLEZ (FINALES DEL SIGLO XIX)

Prignano, Ángel, Una Historia de Relieve. Biblioteca Argentina para ciegos. 1924-2004. Ochenta años de servicio, Buenos Aires, Biblioteca Argentina para Ciegos, 2004.

Varsavsky, Judith A., "El movimiento asociativo de los ciegos en América Latina", Cadernos GESTA, 1 (julho 2001) [en línea], disponible en $<$ http://www.gesta.org/gesta01/> [Consulta: 12/06/ 2014].

Vilanova Rodríguez, Alberto, Los gallegos en la Argentina, Buenos Aires, Ediciones Galicia, 1966.

Villares, Ramón, “La ciudad de los 'Dos Apósteles'(1875-1936)”, en Portela Silva, E. (coord.), Historia de la ciudad de Santiago de Compostela, Santiago de Compostela, Universidad de Santiago de Compostela, 2003, págs. 465-480. 
